\title{
Relationship of Scratch Test and Glenohumeral Internal Rotation and Horizontal Adduction Range of Motion
}

\author{
Min-Hyeok Kang, PT, Ph.D \\ Department of Physical Therapy, International University of Korea, Jinju, South Korea
}

Background To identify limitation of combined shoulder movements, the scratch test has been widely used in clinical settings. However, no study has examined the relationship between the scratch test and glenohumeral (GH) joint passive range of motion (PROM).

Purpose To investigate the relationship between the scratch test and GH internal rotation (IR) and horizontal adduction (HA) PROM.

Study design Cross-sectional study.

Methods Twenty-four healthy subjects were recruited in this study. Bilateral GH IR and HA PROM were measured using a smartphone application in supine position with $90^{\circ}$ of shoulder and elbow flexion. Value of the scratch test was measured using tape measures. Pearson product moment correlations were performed to identify correlation between the scratch test and GH IR and HA PROM.

Results Significant correlation between the scratch test and GH IR PROM $(p=0.004, r=0.408$ and GH HA PROM $(p<0.001, r=0.620)$ was revealed.

Conclusions These findings suggest that the scratch test could be helpful as well as quick measurement to identify GH joint ROM.

Key words Horizontal adduction; Internal rotation; Scratch test.

JMST

2018; 2(2): 29-32

Published Online

Dec 31, 2018

pISSN 2635-8573

eISSN 2635-8581

\section{Article History}

Received 17 September 2018

Revised 19 September 2018 (1st)

Revised 12 October 2018

(2nd)

Accepted 18 October 2018

\section{CONTACT}

kmhyuk01@gmail.com

Min-Hyeok Kang, Department of Physical-

Therapy, International

University of Korea,

Jinju, South Korea

\section{This is an Open-Access article distributed under the terms of the Creative Commons Att- ribution Non-Commercial Li- cense (http://creativecommons org/licenses/by-nc/4.0) which permits unrestricted non-co- mmercial use, distribution, and reproduction in any me- dium, provided the origina} work is properly cited.

\section{INTRODUCTION}

Limitation of shoulder range of motion (ROM) is associated with various shoulder injuries. ${ }^{1-3}$ Limited shoulder ROM is caused by tightness of muscles surrounding shoulder joint and glenohumeral (GH) joint capsule tightness. $^{3-5}$ Especially, it has been reported limited GH internal rotation (IR) ROM due to tightness of posterior shoulder tissues leads to shoulder impingement and shoulder labrum lesion. ${ }^{1-3}$ In addition, tightness of posterior shoulder tissues induce anterior translation of humeral head during GH horizontal adduction (HA), that leads to abnormal arthrokinematics of GH joint. ${ }^{6}$ So, GH IR and HA passive ROM (PROM) is often measured to identify causes of shoulder injuries in clinical settings.

Measures of GH IR PROM are generally performed in supine position with fixation of scapula for accurate measures. $^{4,7-9}$ Similarly, GH HA PROM is measured while fixing scapular lateral boarder to limit unnecessary scapular movement. ${ }^{10,11}$ These methods facilitate measurement of shoulder ROM accurately; however, these methods could lead to difference in measured value depending on expertise of examiners. The scratch test is another way to measure combined shoulder IR ROM. ${ }^{12}$ During the scratch test, both hands are placed on upper and lower regions of the back, respectively, so combined shoulder IR, adduction, and extension in the lower arm side are measured. ${ }^{12}$ This maneuver has the advantage of facilitating quick and easy 
understanding of limitation of shoulder movement.

To address posterior shoulder tightness, various stretching exercises (e.g., sleeper stretching and cross-body stretching) are performed in clinical settings. Previous studies revealed that GH IR and HA ROM significantly increased after these stretching exercises. ${ }^{5,9,10,13}$ These findings support that GH IR and HA PROM are valid variables to identify posterior shoulder tightness. However, traditional methods of GH IR and HA PROM measures have time constraints. Although the scratch test could identify combined GH IR ROM quickly, no study examined the relationship value of scratch test and GH IR and HA ROM. Only significant correlation between GH IR and HA PROM was identified in previous studies. ${ }^{3,14,15}$ So, the purpose of this study was to identify the relationship between the scratch test and GH IR and HA ROM.

\section{METHODS}

\section{Participants}

In total, 24 university students without shoulder pain (10 males and 14 females; age $=23.00 \pm 2.23$; height $=167.67 \pm$ $7.71 \mathrm{~cm}$; body weight $=64.33 \pm 10.65 \mathrm{~kg}$ ) were recruited in this study. Participants were excluded if they had history of shoulder surgery or shoulder injury in the last year. Participants provided an explanation of procedures and agreed to participate.

Measures of glenohumeral internal rotation range of motion

GH IR ROM was passively measured in supine position with $90^{\circ}$ of flexion of shoulder and elbow in the tested side. An examiner passively lowered forearm for GH IR while grapping coracoid process to identify movement of scapular tilt. $^{7,9}$ When an examiner felt scapular movement, passive IR was stopped, and smartphone was placed on the dorsal side of the forearm. The end-range of GH IR angle was measured using the smartphone application of inclinometer (Clinometer-level and slope finder; Plaincode Software Solutions, Stephanskirchen, Germany).

Measures of glenohumeral horizontal adduction range of motion

To measure GH HA PROM, participants were positioned in supine position with $90^{\circ}$ of flexion of shoulder and elbow in the tested side. An examiner gapped and fixed lateral boarder of scapular using thenar eminence to limit unnecessary scapular protraction while moving humerus across the body without humeral rotation. ${ }^{10,11}$ Smartphone was placed on the dorsal side of the humerus at the end-range of $\mathrm{GH}$ $\mathrm{HA}$, and the HA angle was measured using smartphone application of inclinometer.

\section{Scratch test}

To perform the scratch test, participants were asked to place a hand, in the tested side, on the lower region of the back while the other hand was placed on the upper region of the back (Figure 1). ${ }^{12}$ During the scratch test, participants were instructed to clench fists. The distance between two fists was measured using a tape measure.

\section{Data analysis and statistics}

The measures of variables were performed in a random order. Bilateral measures at each variable were repeated three times and mean value of three measurements was used for data analysis. Mean and standard deviation of each variable was calculated using descriptive statistics. Pearson product moment correlations were performed to identify the relationship between the scratch test and GH IR and HA PROM. Magnitude of the relationship was classified as slight $(r<0.25)$, fair $(0.25 \leq r<0.50)$, moderate to good $(0.50 \leq r<0.75)$, and good to excellent $(r \geq 0.75)$. The PASW Statistics 18 (SPSS Inc, Chicago, Illinois) was used as statistical analysis with a level of $p<0.05$.

\section{RESULTS}

The mean \pm standard deviations of GH IR, GH HA, and

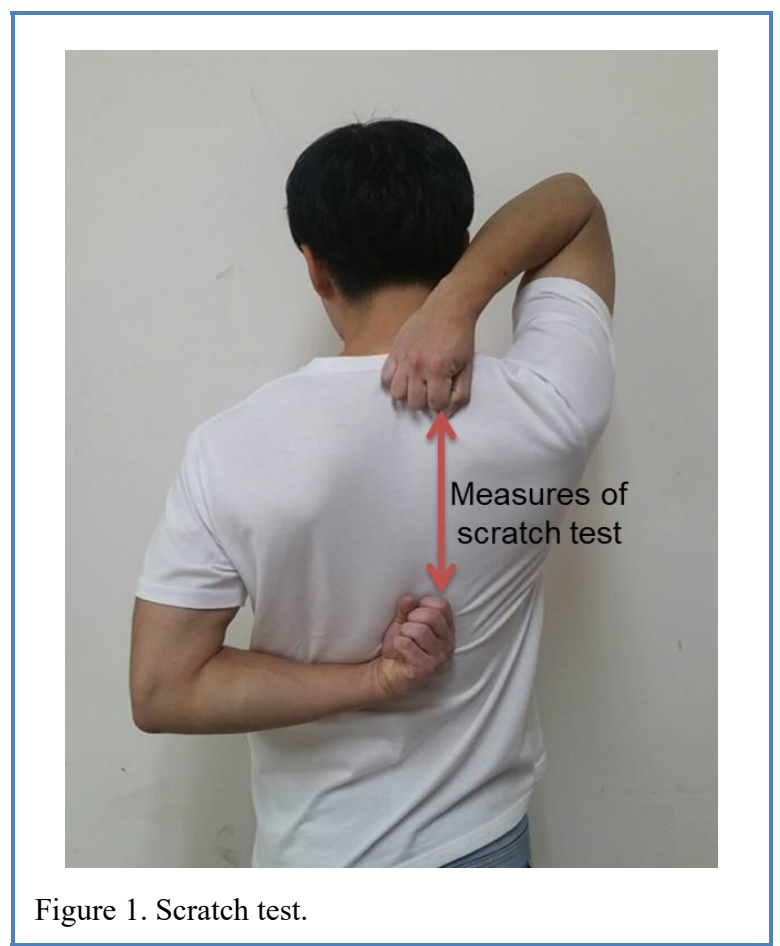


the scratch test were $68.33 \pm 12.49^{\circ}, 34.10 \pm 8.87^{\circ}$, and 18.14 $\pm 9.28 \mathrm{~cm}$, respectively. Value of the scratch test significantly correlated with GH IR PROM $(p=0.004, r=0.408$; Figure 2A) and GH HA PROM ( $p<0.001, r=0.620$; Figure 2B).

\section{DISCUSSION}

Present findings demonstrate that the scratch test has greater relationship with GH HA PROM than GH IR PROM.

A fair correlation between the scratch test and GH IR ROM was affirmed in this study $(r=0.408)$. In this study, GH IR PROM was measured in the position with $90^{\circ}$ of shoulder abduction. This shoulder position increases tension of posterior-inferior portion of GH joint capsule. ${ }^{16,17}$ However, the scratch test was performed from $0^{\circ}$ of shoul-der abduction to position of shoulder adduction, that may rather loosen the inferior GH joint capsule and increase superior GH joint capsule. So, the difference in shoulder position between two tests may influence relatively weak correlation between two variables. Previous findings by McClure et al., ${ }^{13}$ that examine the relationship between value of thumb up the back (TUB) test, the distance between first thoracic spinous process and thumb in the lower back, and GH IR PROM, support our results. Previous findings revealed a fair correlation between TUB test and GH IR PROM $(r=0.44),{ }^{13}$ similar with our findings $(r=0.408)$. Based on previous and present findings, the scratch test may help fairly predict GH IR PROM.

Present findings revealed moderate to good correlation between the scratch test and GH HA ROM $(r=0.620)$. Previous cadaveric study compared change in tension of GH joint capsule between natural condition and contracted joint capsule condition. ${ }^{18}$ The previous study revealed decrease in joint capsule tension by $4 \%$ in the lower region and increase in joint capsule tension by only $4 \%$ in the middle region during $\mathrm{GH}$ HA when the contraction condition was compared to the baseline condition. ${ }^{18}$ Considering that GH IR ROM has significant relationship with posterior capsule tightness ${ }^{3}$ and GH HA ROM, ${ }^{14,15}$ posterior capsule tightness may be also correlated with GH HA ROM. However, contraction of posterior GH joint capsule has minimal effect on tension of middle and lower GH joint capsule during GH HA movements. ${ }^{18}$ So, it is reasonable that GH HA movements may increase tension of superior region of $\mathrm{GH}$ joint capsule. Based on previous findings, tension of superior $\mathrm{GH}$ joint capsule is influenced by position of the scratch test and GH HA movements, that may result in moderate to good relationship between the scratch test and GH HA PROM in this study.

There are limitations in this study. First, the value of scratch test is affected by shoulder IR ROM as well as contralateral shoulder external rotation ROM. Considering that our findings are similar with a previous finding that revealed correlation between GH IR ROM and value of TUB test, ${ }^{13}$ however, the scratch test may reflect limitation of GH IR ROM. Second, only young subjects were recruited in this study. Future studies must include subjects of various ages and subjects with shoulder pain.

\section{CONCLUSION}

This study demonstrates significant correlation between the scratch test and GH IR and HA PROM. Also, the scratch test has moderate to good correlation with GH HA PROM while it has fair correlation with GH IR PROM. These
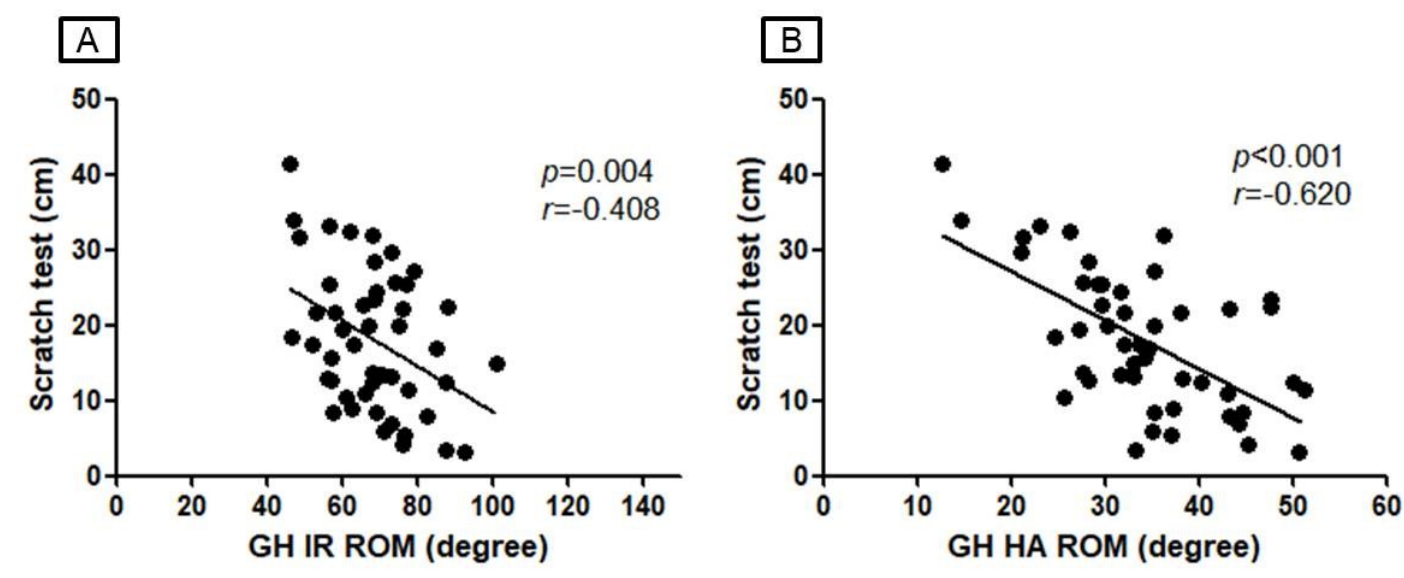

Figure 2. Correlation of scratch test and GH IR ROM (A) and GH HA ROM (B).

Abbreviations: GH, glenohumeral; HA, horizontal adduction; IR, internal rotation; ROM, range of motion. 
findings suggest that the scratch test could be helpful as well as quick measurement to identify GH joint ROM.

\section{Key Points}

Question Are there significant correlation between scratch test and glenohumeral internal rotation and horizontal adduction range of motion?

Findings Scratch test is significantly correlated with both glenohumeral internal rotation and horizontal adduction range of motion, while greater correlation between scratch test and glenohumeral horizontal adduction is found.

Meaning Scratch test could be a useful quick measurement to identify glenohumeral joint range of motion.

\section{Article information}

Conflict of Interest Disclosures: None.

Funding/Support: None.

Acknowledgment: This work was supported by International University of Korea's research fund in 2018.

\section{REFERENCES}

1. Grossman MG, Tibone JE, McGarry MH, Schneider DJ, Veneziani S, Lee TQ. A cadaveric model of the throwing shoulder: A possible etiology of superior labrum anterior-to-posterior lesions. J Bone Joint Surg Am. 2005; 87(4):824-831.

2. Ludewig PM, Cook TM. Translations of the humerus in persons with shoulder impingement symptoms. J Orthop Sports Phys Ther. 2002;32(6):248-259.

3. Tyler TF, Nicholas SJ, Roy T, Gleim GW. Quantification of posterior capsule tightness and motion loss in patients with shoulder impingement. Am J Sports Med. 2000;28(5):668-673.

4. Bailey LB, Shanley E, Hawkins R, et al. Mechanisms of shoulder range of motion deficits in asymptomatic baseball players. Am J Sports Med. 2015;43(11):2783-2793.

5. Yamauchi T, Hasegawa S, Nakamura M, et al. Effects of two stretching methods on shoulder range of motion and muscle stiffness in baseball players with posterior shoulder tightness: A randomized controlled trial. J Shoulder Elbow Surg. 2016;25(9):1395-1403.

6. Harryman DT 2nd, Sidles JA, Clark JM, McQuade KJ, Gibb TD, Matsen FA 3rd. Translation of the humeral head on the glenoid with passive glenohumeral motion. $J$ Bone Joint Surg Am. 1990;72(9):1334-1343.

7. Cools AM, Johansson FR, Cagnie B, Cambier DC, Witvrouw EE. Stretching the posterior shoulder struc- tures in subjects with internal rotation deficit: comparison of two stretching techniques. Shoulder Elbow. 2012;4(1):56-63.

8. Salamh PA, Kolber MJ, Hanney WJ. Effect of scapular stabilization during horizontal adduction stretching on passive internal rotation and posterior shoulder tightness in young women volleyball athletes: A randomized controlled trial. Arch Phys Med Rehabil. 2015;96(2):349356.

9. Tyler TF, Nicholas SJ, Lee SJ, Mullaney M, McHugh MP. Correction of posterior shoulder tightness is associated with symptom resolution in patients with internal impingement. Am J Sports Med. 2010;38(1):114-119.

10. Laudner KG, Sipes RC, Wilson JT. The acute effects of sleeper stretches on shoulder range of motion. J Athl Train. 2008;43(4):359-363.

11. Park KN, Kwon OY, Weon JH, Choung SD, Kim SH. Comparison of the effects of local cryotherapy and passive cross-body stretch on extensibility in subjects with posterior shoulder tightness. J Sports Sci Med. 2014;13(1):84-90.

12. Magee DJ. Orthopedic physical assessment. 6th ed. St. Louis, MO, Elsevier; 2014.

13. McClure P, Balaicuis J, Heiland D, Broersma ME, Thorndike CK, Wood A. A randomized controlled comparison of stretching procedures for posterior shoulder tightness. J Orthop Sports Phys Ther. 2007;37(3):108114.

14. Tyler TF, Roy T, Nicholas SJ, Gleim GW. Reliability and validity of a new method of measuring posterior shoulder tightness. J Orthop Sports Phys Ther. 1999 May;29(5):262-269; discussion 270-274.

15. Laudner KG, Stanek JM, Meister K. Assessing posterior shoulder contracture: The reliability and validity of measuring glenohumeral joint horizontal adduction. $J$ Athl Train. 2006;41(4):375-380.

16. Gagey OJ, Boisrenoult P. Shoulder capsule shrinkage and consequences on shoulder movements. Clin Orthop Relat Res. 2004;(419):218-222.

17. Gerber C, Werner CM, Macy JC, Jacob HA, Nyffeler RW. Effect of selective capsulorrhaphy on the passive range of motion of the glenohumeral joint. J Bone Joint Surg Am. 2003;85-A(1):48-55.

18. Borstad JD, Dashottar A. Quantifying strain on posterior shoulder tissues during 5 simulated clinical tests: A cadaver study. J Orthop Sports Phys Ther. 2011; 41(2):90-99. 